\title{
Entrance channels and alpha decay half-lives of the heaviest elements
}

\author{
G. Royer, K. Zbiri, C. Bonilla ${ }^{1}$ \\ Laboratoire Subatech, UMR: IN2P3/CNRS-Université-Ecole des Mines, \\ 4 rue A. Kastler, 44307 Nantes Cedex 03, France
}

\begin{abstract}
The barriers standing against the formation of superheavy elements and their consecutive $\alpha$ decay have been determined in the quasimolecular shape path within a Generalized Liquid Drop Model including the proximity effects between nucleons in a neck, the mass and charge asymmetry, a precise nuclear radius and the shell effects given by the Droplet Model. For moderately asymmetric reactions doublehump potential barriers stand and fast fission of compact shapes in the outer well is possible. Very asymmetric reactions lead to one hump barriers which can be passed only with a high energy relatively to the superheavy element energy. Then, only the emission of several neutrons or an $\alpha$ particle can allow to reach an eventual ground state. For almost symmetric heavy-ion reactions, there is no more external well and the inner barrier is higher than the outer one. Predictions for partial $\alpha$ decay half-lives are given.
\end{abstract}

PACS: 25.70.Jj; 21.60.Ev; 23.60.+e

Keywords: Superheavy nuclei; Fusion; Alpha decay; Liquid Drop Model.

$\overline{1}$ E-mail:royer@subatech.in2p3.fr

Preprint submitted to Elsevier Science 15 November 2018 


\section{Introduction}

Using heavy-ion reactions of mean asymmetry ( $\mathrm{Ni}$ on $\mathrm{Bi}$ and $\mathrm{Zn}$ on $\mathrm{Pb}$ $[1,2]$ ) and more recently highly asymmetric reactions ( $\mathrm{Ca}$ on $\mathrm{U}, \mathrm{Pu}$ and $\mathrm{Cm}$ $[3,4,5])$ new very heavy elements have been synthetized. In these very long and difficult experiments the number of detected events ( $\alpha$ decay chains) is very low and the interpretation of the primary experimental data is discussed $[6,7,8]$. Actually, it is assumed that the lower limit for the fission barrier heights of these heaviest elements is around $6 \mathrm{MeV}$ [9]. To form other superheavy nuclei, many other reactions covering all the asymmetry range are planned or are proposed using other isotopes, inverse kinematics and radioactive nuclear beams (and targets) $[10,11,12]$.

The purpose of the present work is, firstly, to study the potential barriers standing in the quasimolecular shape path for these new suggested entrance channels and to look at their dependence on the next proton magic number $(114,120$ or 126). The potential barriers relative to already realized experiments have been determined in a precedent paper [13]. The second objective is to determine the partial $\alpha$ decay half-lives of these superheavy elements from the $\mathrm{Q}$ values given by a recent version of the Thomas-Fermi model [14].

The Generalized Liquiq Drop Model which has allowed to describe most of the fusion [15], fission [16,17], light nucleus [18] and $\alpha$ emission [19] data has been used once again. The shell effects have been derived from the Droplet Model formulae [20].

\section{Quasimolecular shapes}

To describe the entrance channel leading from two spheres to one sphere and the alpha emission a shape sequence leading rapidly to the formation of a deep neck while keeping almost spherical ends has been retained (see Fig. 1). Mathematically, these quasimolecular shapes correspond to two joined elliptic lemniscatoids [15] assuming volume conservation. Analytic formulae are available for the main shape-dependent functions : volume, surface, distance between mass centres and moment of inertia. Similar compact shapes have been also adopted to determine the energy of superheavy nuclei [21]. For the exit channel these shapes implicitly suppose that the $\alpha$ particle is rather formed at the surface of the parent nucleus. 


\section{Generalized Liquid Drop Model}

The macroscopic energy is determined within the Generalized Liquid Drop Model previously defined and used to describe the fusion and fission processes as well as the light nucleus and $\alpha$ emissions $[15,16,17,18,19]$.

$$
E=E_{\text {Vol }}+E_{\text {Surf }}+E_{\text {Coulomb }}+E_{\text {Prox }} .
$$

When the colliding nuclei or the spherical fragments are separated:

$$
\begin{aligned}
& E_{\text {Vol }}=-15.494\left[\left(1-1.8 I_{1}^{2}\right) A_{1}+\left(1-1.8 I_{2}^{2}\right) A_{2}\right] M e V, \\
& E_{\text {Surf }}=17.9439\left[\left(1-2.6 I_{1}^{2}\right) A_{1}^{2 / 3}+\left(1-2.6 I_{2}^{2}\right) A_{2}^{2 / 3}\right] M e V, \\
& E_{\text {Coulomb }}=0.6 e^{2} Z_{1}^{2} / R_{1}+0.6 e^{2} Z_{2}^{2} / R_{2}+e^{2} Z_{1} Z_{2} / r,
\end{aligned}
$$

where $A_{i}, Z_{i}, R_{i}$ and $I_{i}$ are the masses, charges, radii and relative neutron excesses of the two nuclei. $\mathrm{r}$ is the distance between the mass centres.

The radii $R_{i}$ are given by:

$$
R_{i}=\left(1.28 A_{i}^{1 / 3}-0.76+0.8 A_{i}^{-1 / 3}\right) f m .
$$

This formula allows to follow the experimentally observed increase of the ratio $r_{i}=R_{i} / A_{i}^{1 / 3}$ with the mass; for example, $r_{0}=1.13 \mathrm{fm}$ for ${ }^{48} \mathrm{Ca}$ and $r_{0}=$ $1.18 \mathrm{fm}$ for ${ }^{248} \mathrm{Cm}$.

For one-body shapes, the volume, surface and Coulomb energies are defined as

$$
\begin{aligned}
& E_{\text {Vol }}=-15.494\left(1-1.8 I^{2}\right) A M e V, \\
& E_{\text {Surf }}=17.9439\left(1-2.6 I^{2}\right) A^{2 / 3}\left(S / 4 \pi R_{0}^{2}\right) M e V, \\
& E_{\text {Coulomb }}=0.6 e^{2}\left(Z^{2} / R_{0}\right) \times 0.5 \int\left(V(\theta) / V_{0}\right)\left(R(\theta) / R_{0}\right)^{3} \sin \theta d \theta .
\end{aligned}
$$

$I$ is the relative neutron excess and $S$ the surface of the one-body deformed nucleus. $V(\theta)$ is the electrostatic potential at the surface and $V_{0}$ the surface potential of the sphere. The radius of the compound nuclear system has been calculated from the radii of the two fragments assuming volume conservation.

The surface energy results from the effects of the surface tension forces in a half space. When there are nucleons in regard in a neck or a gap between colliding nuclei or separated fragments an additional term called proximity 
energy must be added to take into account the effects of the nuclear forces between the close surfaces in regard. This term is essential to describe smoothly the two-body to one-body transition and to obtain reasonable fusion barrier heights. It moves the barrier top to an external position and strongly decreases the pure Coulomb barrier.

$$
E_{\text {Prox }}(r)=2 \gamma \int_{h_{\min }}^{h_{\max }} \Phi[D(r, h) / b] 2 \pi h d h,
$$

where $h$ is the distance varying from the neck radius or zero to the height of the neck border. $D$ is the distance between the surfaces in regard and $b=0.99 \mathrm{fm}$ the surface width. $\Phi$ is the proximity function of Feldmeier [22]. The surface parameter $\gamma$ is the geometric mean between the surface parameters of the two nuclei or fragments:

$$
\gamma=0.9517 \sqrt{\left(1-2.6 I_{1}^{2}\right)\left(1-2.6 I_{2}^{2}\right)} M_{e V ~ f m}^{-2}
$$

In this GLDM the surface diffuseness is not taken into account and the proximity energy vanishes when there is no neck or gap. The combination of these GLDM and shape sequence has allowed to reproduce the fusion barrier heights and radii, the fission and the $\alpha$ and cluster radioactivity data.

\section{Potential barriers governing the entrance channel}

The macroscopic potential barriers (deformation energy) standing in front of some heavy-ion combinations leading to ${ }^{270} 110$ and ${ }^{302} 120$ compound systems are displayed in Fig. 2. Some reactions using very exotic nuclei are not still feasible. Within this GLDM the nuclear proximity energy introduces an inflection in the curve and the outer barrier top corresponds always to an unstable equilibrium between the repulsive Coulomb forces and the attractive proximity forces for two separated nuclei. For the highest asymmetries the external fusion barrier energy is higher than the spherical system energy and a potential pocket appears after crossing the barrier. In this description, the nuclear system can reach a quasi-spherical shape with little excitation energy via a tunneling process. This does not prove the stability of the formed system. For the exit channel all the symmetric and asymmetric fission and $\alpha$ emission barriers must be investigated. With decreasing asymmetry the outer well progressively disappears as the outer peak. It will be very difficult to reach the eventual ground state of the superheavy element via these almost symmetric reactions. Predictions from such barriers for four asymmetric and almost symmetric reactions leading to ${ }^{246} \mathrm{Fm}$ had been compared in a previous paper [15] 
with experimental data on evaporation residues and fast fission events and the agreement was very good.

In ordinary fusion studies, it is only the preceding barriers which are taken into account. The macroscopic energy does not reproduce very accurately the $\mathrm{Q}$ value since the nonuniform distribution of single-particle levels leading to the shell correction term and pairing energy is not taken into account as also Wigner energy,... In Fig. 3, the difference between the theoretical $\mathrm{Q}$ value given by the Thomas-Fermi model [14] and the GLDM one has been empirically added at the macroscopic energy of the compound nucleus with a linear (in r) attenuation factor vanishing at the contact point. Such a description is valid if the nuclear system has enough time to relax and built its shells and pairs during its descent to a quasi-spherical shape. Then the barrier against reseparation is higher and the external minimum disappears for the highest asymmetries.

In the following the shell corrections proposed in the Droplet Model $[20,16]$ will be used and the formulae are recalled here.

$$
E_{\text {Shell }}=E_{\text {Shell }}^{\text {sphere }}\left(1-2 \theta^{2}\right) e^{-\theta^{2}}
$$

The shell corrections for a spherical nucleus are given by

$$
E_{\text {Shell }}^{\text {sphere }}=5.8\left[(F(N)+F(Z)) /(0.5 A)^{2 / 3}-0.26 A^{1 / 3}\right] M e V
$$

where, for $M_{i-1}<X<M_{i}$,

$$
F(X)=q_{i}\left(X-M_{i-1}\right)-0.6\left(X^{5 / 3}-M_{i-1}^{5 / 3}\right)
$$

$M_{i}$ are the magic numbers and

$$
q_{i}=0.6\left(M_{i}^{5 / 3}-M_{i-1}^{5 / 3}\right) /\left(M_{i}-M_{i-1}\right) .
$$

Within this algebraic approach it is possible to study the influence of the selected value for the highest proton magic number. For the two highest neutron magic numbers the values 126 and 184 have been retained.

$$
\theta^{2}=(\delta R)^{2} / a^{2}
$$

The distortion $\theta a$ is the root mean square of the deviation of the nuclear surface from the sphere, a quantity which incorporates indiscriminately all types of deformation. The range a has been chosen to be $0.32 r_{0}$. 
Potential barriers for three very asymmetric reactions ('warm' reactions) ${ }^{48} \mathrm{Ca}+{ }^{244} \mathrm{Pu},{ }^{48} \mathrm{Ca}+{ }^{248} \mathrm{Cm}$ and ${ }^{50} \mathrm{Ti}+{ }^{248} \mathrm{Cm}$ are shown in Fig. 4 assuming different hypotheses. Due to the high asymmetry and, consequently, to a low Coulomb repulsion and proximity energy the barrier against reseparation is wide and high. There is no double-hump barriers. Even for a subbarrier tunneling of 6 or $7 \mathrm{MeV}$ in the entrance channel and even if the shells and pairs have not enough time to develop (dashed curve) before reaching the quasispherical shape the nuclear system has enough energy to reach this quasispherical compound system. The excitation energy relatively to the ground state energy is more than $30 \mathrm{MeV}$, allowing the emission of several neutrons or an $\alpha$ particle. The different hypotheses on the proton magic number do not change the global predictions in the entrance path.

Potential barriers governing the medium asymmetry reactions ('cold' reactions) ${ }^{64} \mathrm{Ni}+{ }^{208} \mathrm{~Pb},{ }^{70} \mathrm{Zn}+{ }^{208} \mathrm{~Pb},{ }^{76} \mathrm{Ge}+{ }^{208} \mathrm{~Pb}$ and ${ }^{82} \mathrm{Se}+{ }^{208} \mathrm{~Pb}$ are displayed in Fig. 5. A wide macroscopic potential pocket due mainly to higher proximity energy and Coulomb repulsion (relatively to warm reactions) appears at large deformations. Whatever the microscopic correction assumptions are double-hump barriers begin to appear for the $\mathrm{Ni}$ on $\mathrm{Pb}$ and $\mathrm{Zn}$ on $\mathrm{Pb}$ reactions. Experimentally the beam energy was respectively 239 and $257 \mathrm{MeV}$. Consequently, these successfull experiments correspond to subbarrier fusion processes in our approach and the quasispherical system can be reached by tunneling even if the shells and pairs are not completely built. For the two last reactions the inner macroscopic hill is higher than the external barrier. Incomplete fusion and fast fission events in the external pocket are the main exit channels since the neck between the two nuclei is formed and exchanges of nucleons may occur. If the reorganization of the single-particle levels is very rapid then the value of the proton magic number begins to play some role. So an open question is whether at large deformations the nucleon shells can take form to stabilize the nuclear system before investigating a peculiar exit channel. The pre or post equilibrium nature of the evaporation process of the excess neutron is also crucial.

The same comments are valid for the two cold reactions shown in Fig. 6 leading to nuclei of charge 118 and 119. A value of 120 for the next proton magic number naturally would slightly increase the possibility to form such superheavy elements. For still heavier mass and charge and more symmetric heavy-ion reactions (see Fig. 7) the potential pocket between the two peaks progressively disappears. A tunneling effect in the outer peak does not allow to reach a quasispherical state. Only a subbarrier fusion through the inner peak could eventually lead to a compound nucleus if the microscopic contributions grow rapidly at the beginning of the fusion process and if the charge of the compound system is the next proton magic number.

The symmetric decay barriers for ${ }^{283} 112,{ }^{292} 116,{ }^{295} 120$ and ${ }^{311} 126$ are 
given in Fig. 8. The microscopic contributions play the main role. The height and width of the one hump fission barrier is governed by the proximity of a proton or neutron magic number. The barrier height still reaches several $\mathrm{MeV}$ and can reach $10 \mathrm{MeV}$ if the neutron and proton shell effects add each other.

The characteristics of the macroscopic potential barriers for the new suggested reactions $[10,11,12]$ leading possibly to superheavy elements are shown in table 1. The Q value is extracted from [14]. The energy of the external peak relatively to the sphere energy is positive for $Z_{1} Z_{2}$ lower than around 2500 . The position of the external barrier corresponds to the contact point of the colliding nuclei when $Z_{1} Z_{2}$ is higher than around 2600. There is no more real external macroscopic barrier for $Z_{1} Z_{2}$ higher than around 3200 .

In the hope of reaching the most efficient beam energy, formulas giving the l-dependent fusion barrier height $E_{f u s}$ and radius $R_{f u s}$ are proposed.

$$
\begin{aligned}
E_{f u s, l}(\mathrm{MeV}) & =E_{\text {fus }, l=0} \\
& +\frac{l(l+1)}{0.02081\left(A_{1}^{5 / 3}+A_{2}^{5 / 3}\right)+\frac{0.0506 A_{1} A_{2}\left(A_{1}^{1 / 3}+A_{2}^{1 / 3}\right)^{2}\left(1.908+\frac{3.94}{Z_{1} Z_{2}}-0.0857 \ln \left(Z_{1} Z_{2}\right)\right)^{2}}{A_{1}+A_{2}}},
\end{aligned}
$$

with

$$
\begin{aligned}
& E_{\text {fus }, l=0}(\mathrm{MeV})=-19.38+\frac{2.1388 Z_{1} Z_{2}+59.427\left(A_{1}^{1 / 3}+A_{2}^{1 / 3}\right)-27.07 \ln \left(\frac{Z_{1} Z_{2}}{A_{1}^{1 / 3}+A_{2}^{1 / 3}}\right)}{\left(A_{1}^{1 / 3}+A_{2}^{1 / 3}\right)\left(2.97-0.12 \ln \left(Z_{1} Z_{2}\right)\right)},(1, \quad 18) \\
& \begin{aligned}
R_{f u s, l}(\mathrm{fm}) & =\left(A_{1}^{1 / 3}+A_{2}^{1 / 3}\right)\left[1.532+6.48 / Z_{1} Z_{2}\right] \\
& -0.000507\left(A_{1}^{1 / 3}+A_{2}^{1 / 3}\right)^{2} \ln \left(Z_{1} Z_{2}\right)^{2}-\frac{52.8 l^{2}}{A_{1}^{2} A_{2}^{2}}-\frac{1.99 l^{2}}{\left(A_{1}+A_{2}\right)^{2}}
\end{aligned}
\end{aligned}
$$

For frontal collisions the fusion radius is still more precisely given by

$$
R_{f u s, l=0}(f m)=\left(A_{1}^{1 / 3}+A_{2}^{1 / 3}\right)\left[1.908-0.0857 \ln \left(Z_{1} Z_{2}\right)+\frac{3.94}{Z_{1} Z_{2}}\right] .
$$

\section{$5 \quad$ Excitation energy}

Assuming a full equilibrated ground state the experimental excitation energy $E_{e x p}^{*}$ is the sum of the beam energy $E_{c m}$ in the mass centre frame and the $Q_{\text {fusion }}$ value. For the heaviest already investigated experiments, $E_{\text {exp }}^{*}$ is compared, in table 2, with the energy $E_{b a r}^{*}$ needed to pass classically the 
outer fusion barrier peak given by the GLDM. The difference between these two values is given in the last column. The negative sign corresponds in our approach to a tunneling process in the entrance channel. The range of some $\mathrm{MeV}$ below or above the barrier and the barrier profiles are consistent with the very low fusion cross sections and the ability to reach the compound nucleus. Let us recall that the fusion barrier height derived from the GLDM is systematically higher than the Bass barrier height and the fusion radius is smaller [13]. Consequently, for most of the presently used incident energies, the fusion is above the barrier for the Bass model and a subbarrier fusion for the GLDM.

\section{$6 \quad \alpha$ decay half-lives}

To determine the potential barriers against $\alpha$ emission, the $\alpha$ decay has been viewed [19] as a very asymmetric spontaneous fission within the GLDM. The difference between the experimental $Q_{\alpha}$ value or the value predicted by the Thomas-Fermi model [14] and the value given by the GLDM has also been added at the sphere energy with a linear attenuation factor vanishing at

the rupture point. Within such an unified fission model the decay constant of the parent nucleus is simply defined as $\lambda=\nu_{0} P$. The assault frequency $\nu_{0}$ has been chosen as $10^{20} \mathrm{~s}^{-1}$. The barrier penetrability $\mathrm{P}$ has been calculated within the general form of the action integral. Most of the decay path corresponds to two-body shapes and the reduced mass approximation is sufficient for the inertia. The predicted $\alpha$ decay half-lives agree with the experimental data in the whole mass range and also for the known heaviest elements $[8,19]$. Accurate formulae depending only on the mass and charge of the parent nucleus and $Q_{\alpha}$ have been extracted from the data. They are recalled here respectively for the even $(\mathrm{Z})$-even $(\mathrm{N})$, even-odd, odd-even and odd-odd nuclei.

$$
\begin{aligned}
& \log _{10}\left[T_{1 / 2}(s)\right]=-25.31-1.1629 A^{1 / 6} \sqrt{Z}+\frac{1.5864 Z}{\sqrt{Q_{\alpha}}}, \\
& \log _{10}\left[T_{1 / 2}(s)\right]=-26.65-1.0859 A^{1 / 6} \sqrt{Z}+\frac{1.5848 Z}{\sqrt{Q_{\alpha}}} \\
& \log _{10}\left[T_{1 / 2}(s)\right]=-25.68-1.1423 A^{1 / 6} \sqrt{Z}+\frac{1.592 Z}{\sqrt{Q_{\alpha}}} \\
& \log _{10}\left[T_{1 / 2}(s)\right]=-29.48-1.113 A^{1 / 6} \sqrt{Z}+\frac{1.6971 Z}{\sqrt{Q_{\alpha}}} .
\end{aligned}
$$

The global predictions for the $\alpha$ decay half-lives of the heaviest elements within the preceding formulas are given in table 3 . If the $\alpha$ decay mode is 
the main decay mode, then for most of them their half-lives will vary from microsecond to some days. Nevertheless, some nuclei $\left({ }^{319,320} 126\right.$ and ${ }^{317} 124$, i.e Z around 124-126 and N around 193-194) have a very low $Q_{\alpha}$ and consequently a very high $\alpha$ decay half-life. About the same results are obtained within the $Q_{\alpha}$ value given by the finite-range Droplet macroscopic model coupled to the folded-Yukawa single-particle microscopic model [23]. The calculation of the half-lives against symmetric and asymmetric fission is another challenge.

\section{Conclusion}

The barriers preventing the formation of superheavy elements and their consecutive $\alpha$ decay have been determined in the quasimolecular shape path within a Generalized Liquid Drop Model including the proximity effects between nucleons in a neck and the mass and charge asymmetry. The dependence on the asymmetry in the entrance channel, on the proton magic number of the compound system and on the introduction of the $\mathrm{Q}$ value has been studied. The shell effects have been determined within the Droplet Model formulae.

For moderately asymmetric reactions double-hump potential barriers stand and fast fission of compact shapes in the outer well is the main exit channel. Very asymmetric reactions lead to one hump barriers which can be passed only with an energy much higher than the ground state energy of the superheavy element. Then, only emission of several neutrons or an $\alpha$ particle can stabilize the nuclear system and allows to reach a ground state. The formation of superheavy elements via almost symmetric reactions is hardly likely.

In this very heavy mass range the Droplet Model formulae lead to fission barrier heights of several $\mathrm{MeV}$ or even $10 \mathrm{MeV}$ for nuclei close to the proton and neutron magic numbers. The main decay mode being the $\alpha$ decay, the predicted half-life depends on the selected theoretical $Q_{\alpha}$ value. Within the $Q_{\alpha}$ given by the Thomas-Fermi model, partial $\alpha$ decay half-lives are proposed. They agree generally with the known experimental data.

\section{References}

[1] S. Hofmann et al, Z. Phys. A 350 (1995) 281.

[2] S. Hofmann et al, Z. Phys. A 354 (1996) 229.

[3] Yu.Ts. Oganessian et al, Phys. Rev. Lett. 83 (1999) 3154.

[4] Yu.Ts. Oganessian et al, Phys. Rev. C 62 (2000) 041604(R). 
[5] Yu.Ts. Oganessian et al, Phys. Rev. C 63 (2000) 011301(R).

[6] P. Armbruster, E. Phys. J. A 7 (2000) 23.

[7] S. Hofmann and G. Münzenberg, Rev. Mod. Phys. 72 (2000) 733.

[8] W. Loveland et al, Phys. Rev. C 66 (2002) 044617.

[9] M.G. Itkis, Yu.Ts. Oganessian and V.I. Zagrebaev, Phys. Rev. C 65 (2002) 044602 .

[10] Raj K. Gupta, M. Balasubramaniam, G. Münzenberg, W. Greiner and W. Scheid, J. Phys. G 27 (2001) 867.

[11] J. Peter et al, Proc. Int. Conf. on nuclear physics at border lines, World Scientific, Lipari, Italy, 2001, p 257.

[12] S. Kumar, M. Balasubramaniam, Raj K. Gupta, G. Münzenberg and W. Scheid, J. Phys. G 29 (2003) 625.

[13] G. Royer and R.A. Gherghescu, Nucl. Phys. A 699 (2002) 479.

[14] W.D. Myers and W.J. Swiatecki, Nucl. Phys. A 601 (1996) 141.

[15] G. Royer and B. Remaud, Nucl. Phys. A 444 (1985) 477.

[16] G. Royer and B. Remaud, J. Phys. G 10 (1984) 1057.

[17] G. Royer and K. Zbiri, Nucl. Phys. A 697 (2002) 479.

[18] G. Royer, R. Moustabchir, Nucl. Phys. A 683 (2001) 182.

[19] G. Royer, J. Phys. G 26 (2000) 1149.

[20] W.D. Myers, Droplet Model of Atomic Nuclei, Plenum, New York, 1977.

[21] R.A. Gherghescu, D.N. Poenaru and W. Greiner, J. Phys. G 23 (1997) 1715.

[22] H. Feldmeier, $12^{\text {th }}$ Summer School on Nuclear Physics, Mikolajki, Poland, 1979.

[23] P. Möller, J.R. Nix, W.D. Myers and W.J. Swiatecki, At. Data Nucl. Data Tables 59 (1995) 185. 


\section{Figure captions}

Fig. 1. Selected shape sequence to simulate the transition from two-body to one-body shapes and the alpha decay path. The nuclei are spherical when they are separated.

Fig. 2. Macroscopic potential barriers for different reactions leading to the ${ }^{270} 110$ and ${ }^{302} 120$ nuclei. $\mathrm{r}$ is the distance between the mass centres. The vertical bar corresponds to the contact point.

Fig. 3. Same as figure 2 for ${ }^{302} 120$ but an adjustment is done to reproduce the Thomas-Fermi model Q value.

Fig. 4. Potential barriers for the ${ }^{48} \mathrm{Ca}+{ }^{244} \mathrm{Pu},{ }^{48} \mathrm{Ca}+{ }^{248} \mathrm{Cm},{ }^{50} \mathrm{Ti}+{ }^{248} \mathrm{Cm}$ reactions. The dashed curve corresponds to the macroscopic barrier. The barrier given by the dashed and double dotted line incorporates a linear correction from the contact point till the sphere to reproduce the $\mathrm{Q}$ value. The full line, dotted curve and dashed and dotted curve include the shell effects given by the Droplet Model assuming respectively a proton magic number of 114, 120 and 126 and an adjustment to reproduce the $\mathrm{Q}$ value.

Fig. 5. Same as figure 4 but for the reactions ${ }^{64} \mathrm{Ni}+{ }^{208} \mathrm{~Pb},{ }^{70} \mathrm{Zn}+{ }^{208} \mathrm{~Pb}$, ${ }^{76} \mathrm{Ge}+{ }^{208} \mathrm{~Pb}$ and ${ }^{82} \mathrm{Se}+{ }^{208} \mathrm{~Pb}$.

Fig. 6. Same as figure 4 but for the reactions ${ }^{86} K r+{ }^{208} \mathrm{~Pb}$ and ${ }^{87} \mathrm{Rb}+{ }^{208} \mathrm{~Pb}$.

Fig. 7. Same as figure 4 but for the reactions ${ }^{88} \mathrm{Sr}+{ }^{208} \mathrm{~Pb},{ }^{116} \mathrm{Cd}+{ }^{181} \mathrm{~W}$ and ${ }^{104} R u+{ }^{208} \mathrm{~Pb}$.

Fig. 8. Symmetric fission barriers for ${ }^{283} 112,{ }^{292} 116,{ }^{295} 120$ and ${ }^{311} 126$. The full line, dotted curve and dashed and dotted curve include the shell effects given by the Droplet Model assuming respectively a proton magic number of 114, 120 and 126; the neutron magic numbers being 126 and 184 .

\section{Table captions}

Table 1. Characteristics of the macroscopic potential barriers for the heaviest nuclear systems. $R_{\min }, R_{e x t}, H_{\min }$ and $H_{\text {ext }}$ are respectively the positions and energies relatively to the sphere of the outer minimum and external maximum of the barrier. $R_{12}$ is the distance between the mass centres at the contact point. $H_{\text {ext,fus }}$ is the GLDM external fusion barrier height.

Table 2. Comparison between the experimental excitation energy $E_{\text {exp }}^{*}$ and the classical excitation energy $E_{b a r}^{*}$ at the top of the GLDM barrier. In the last column the positive or negative sign of the difference $E_{e x p}^{*}-E_{b a r}^{*}$ indicates a fusion above or below the barrier. 
Table 3. Predicted $\log _{10}\left[T_{1 / 2}(s)\right]$ for the heaviest elements and nuclear systems versus the charge and mass of the parent nucleus and the $Q_{\alpha}$ value given by a recent version of the Thomas-Fermi model [14]. 

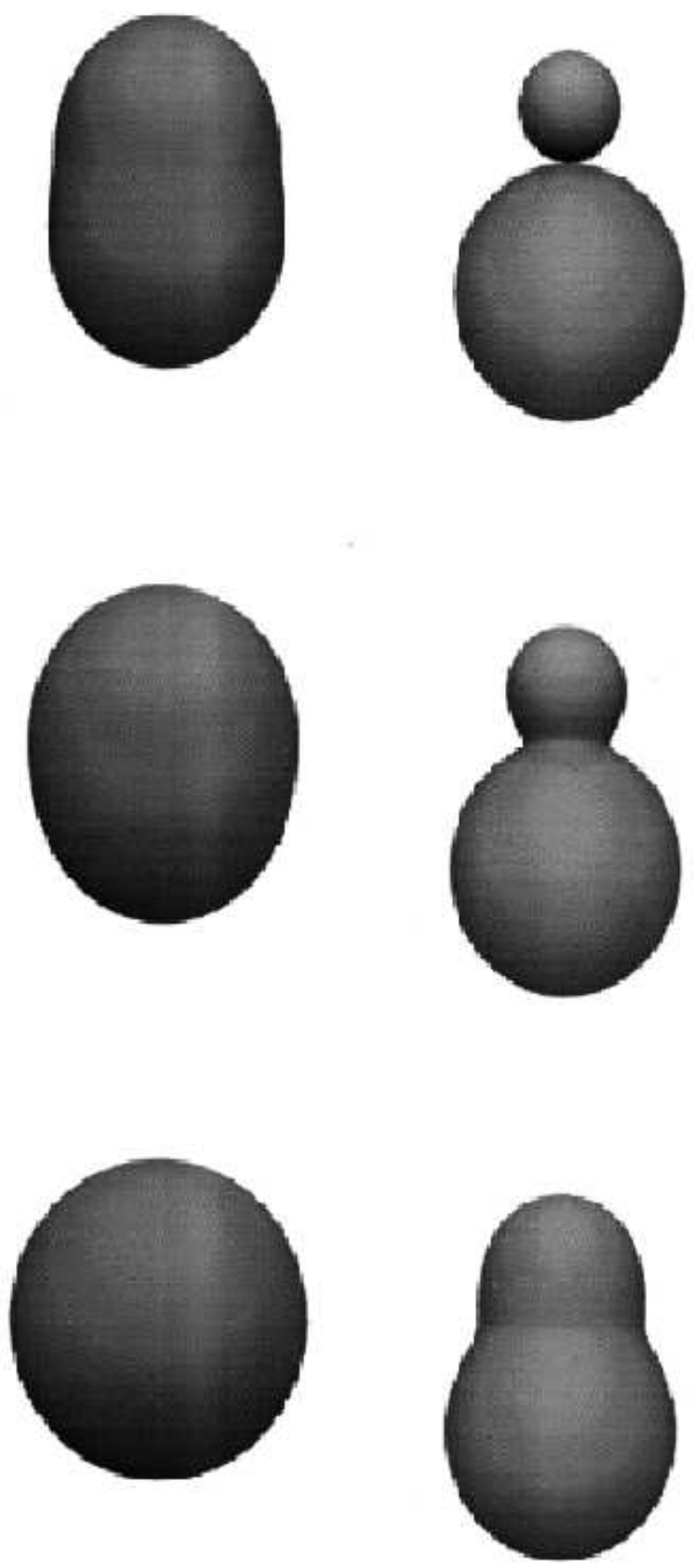


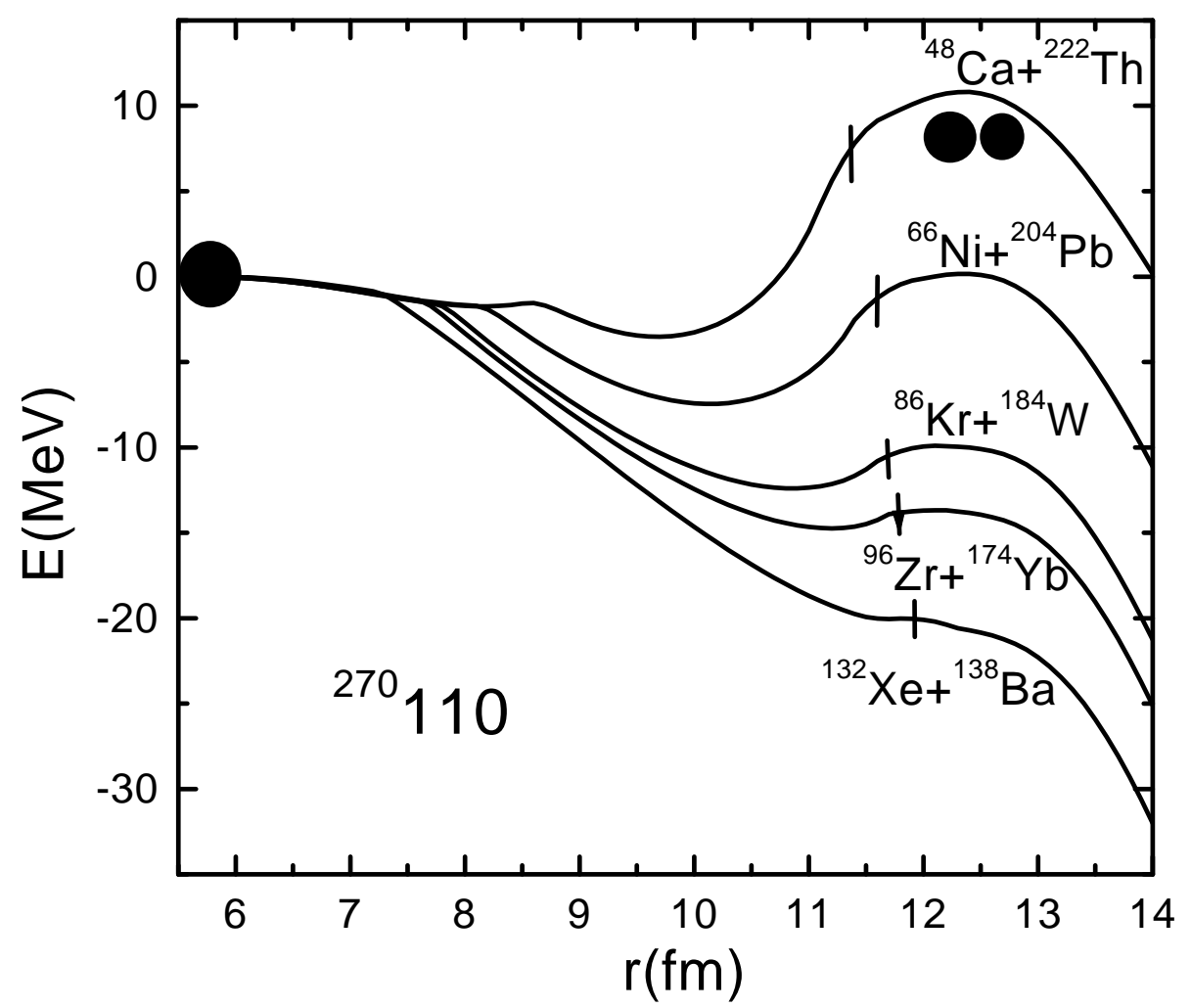




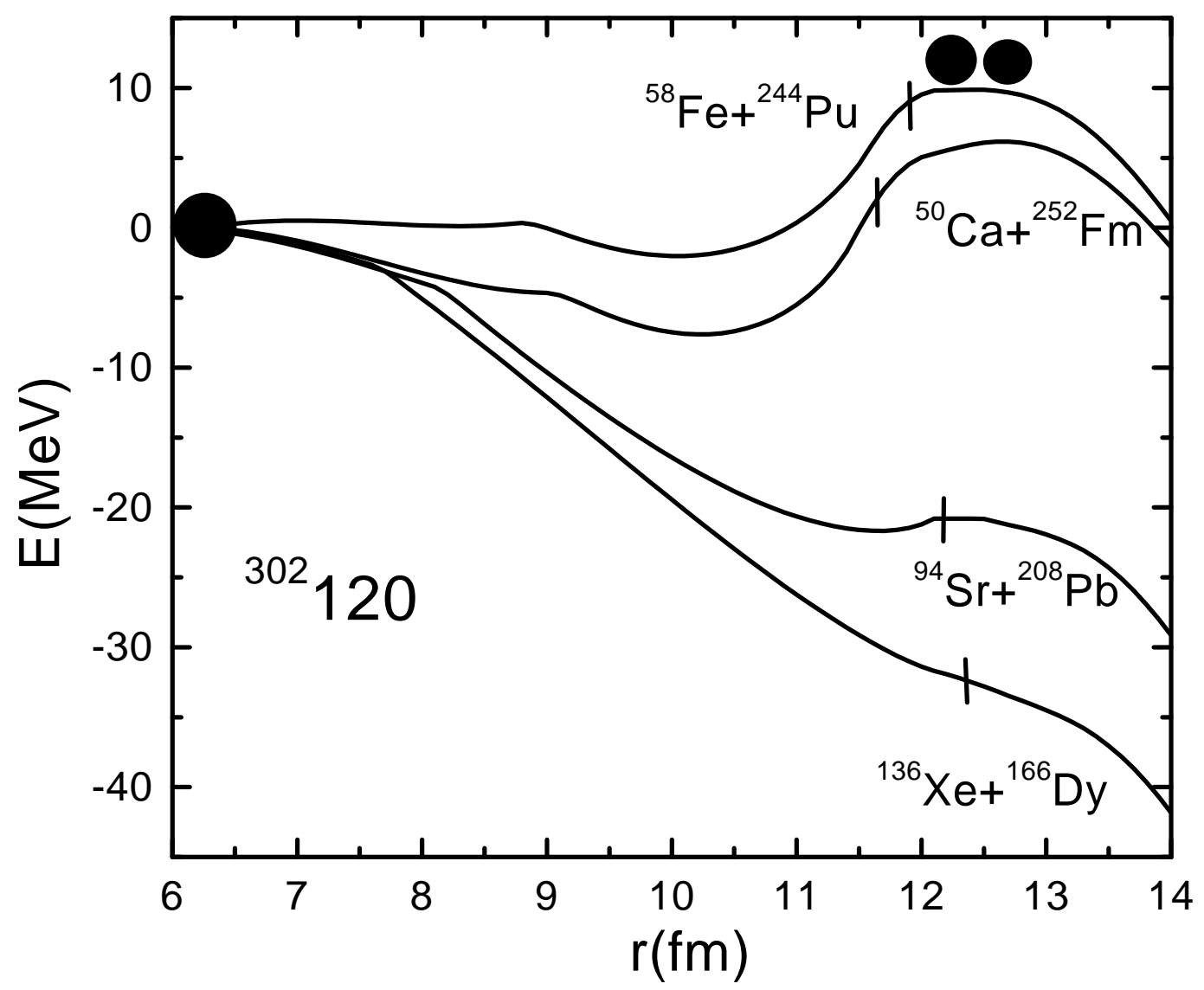




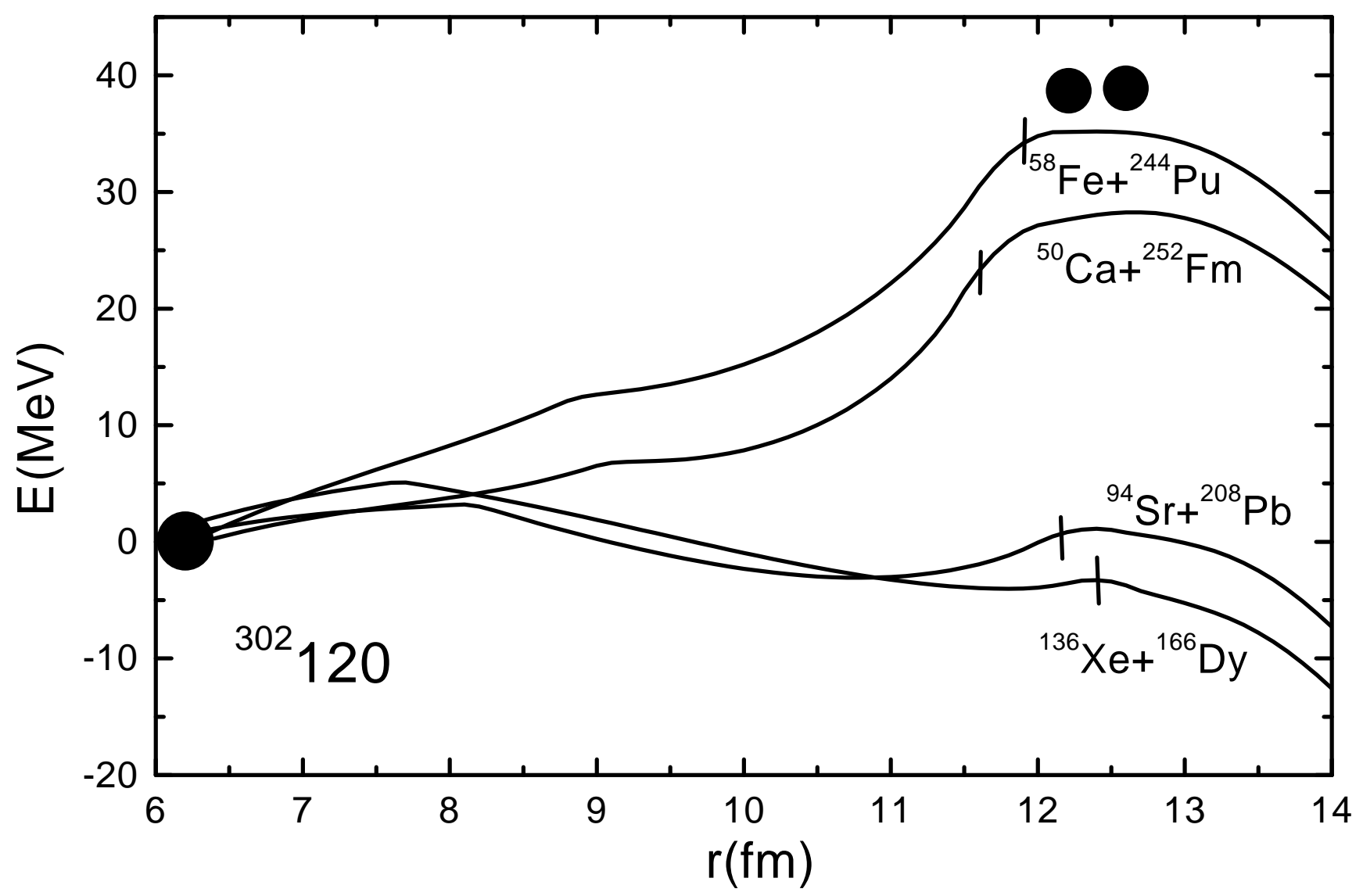




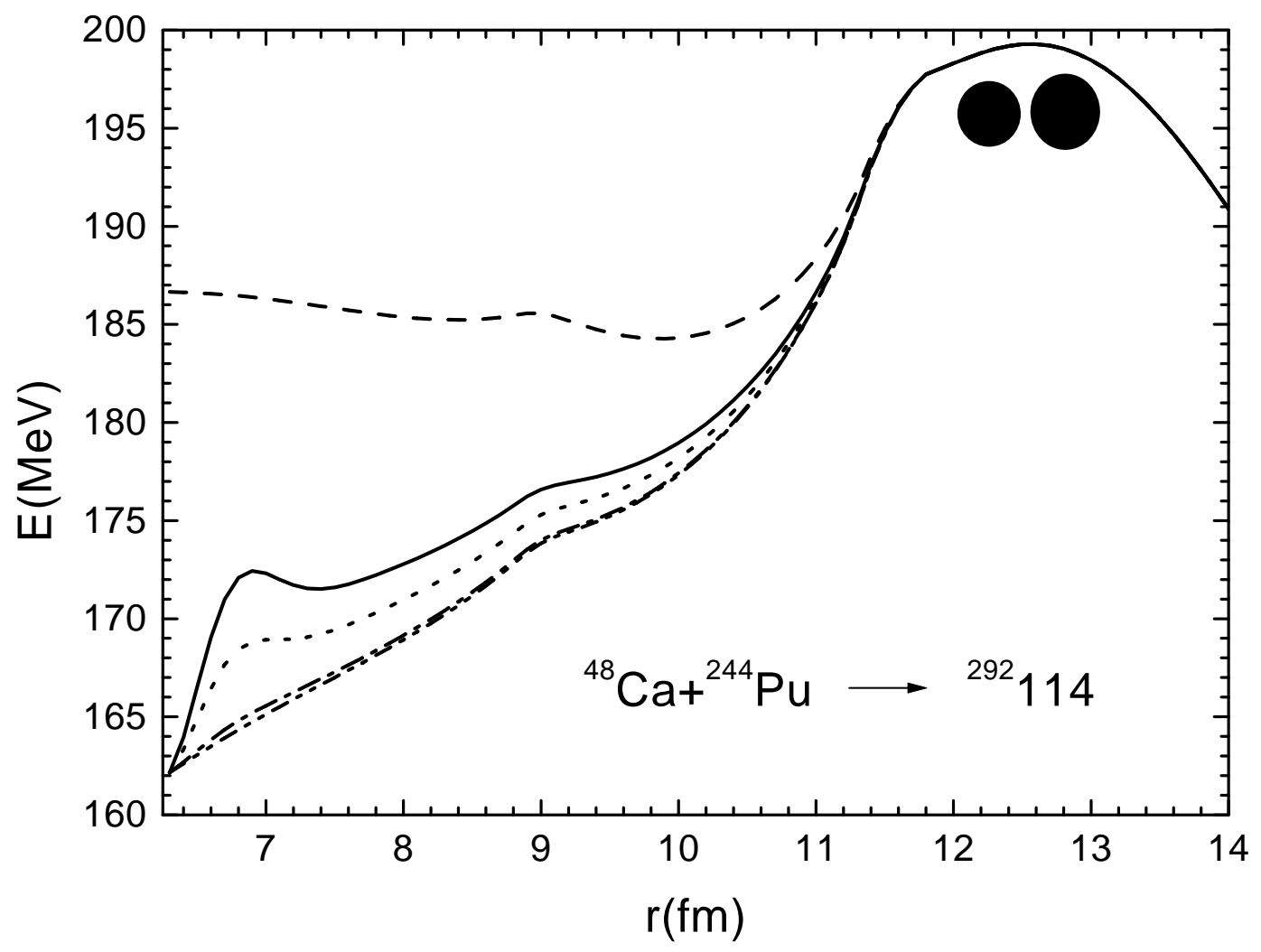




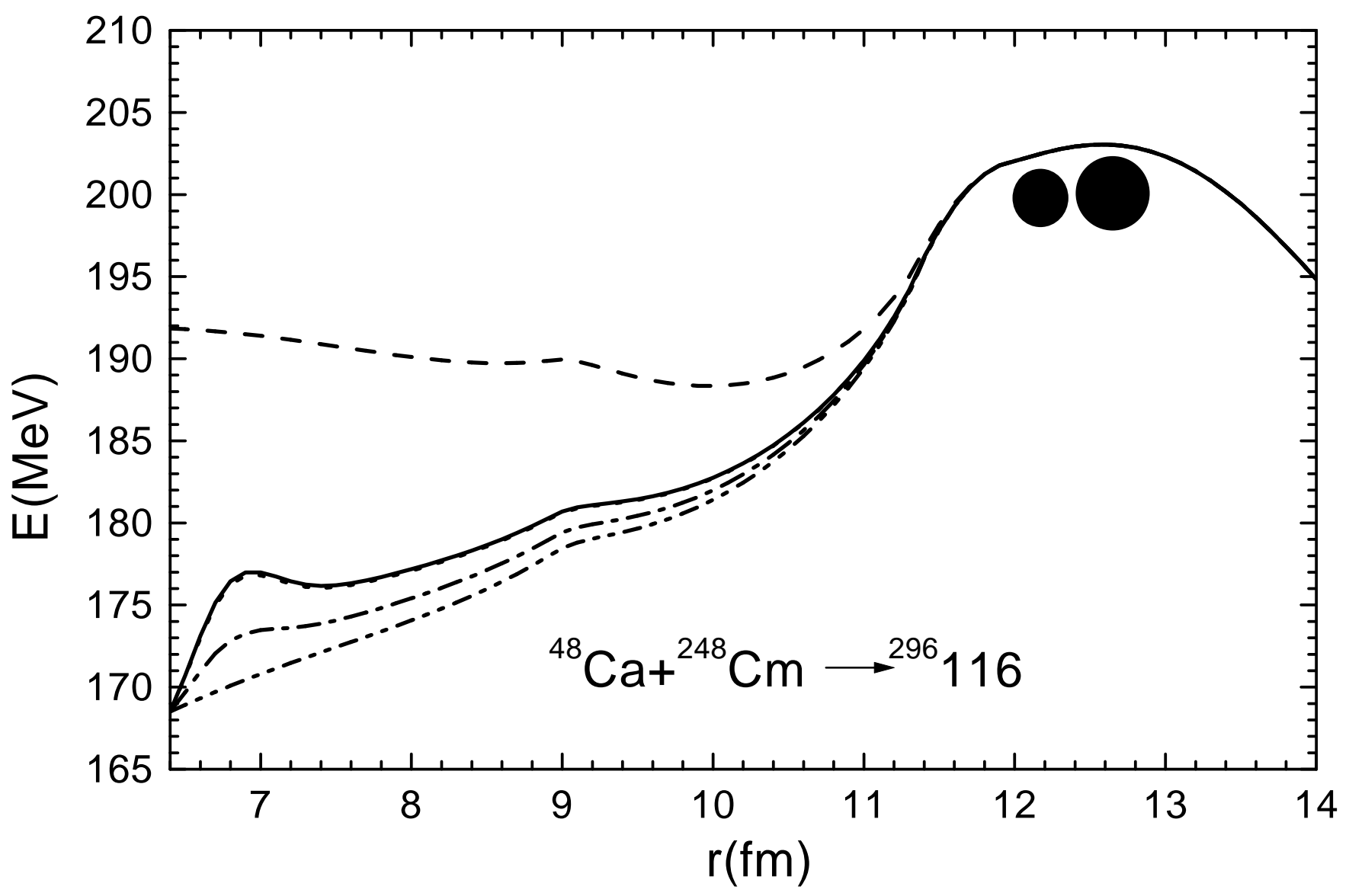




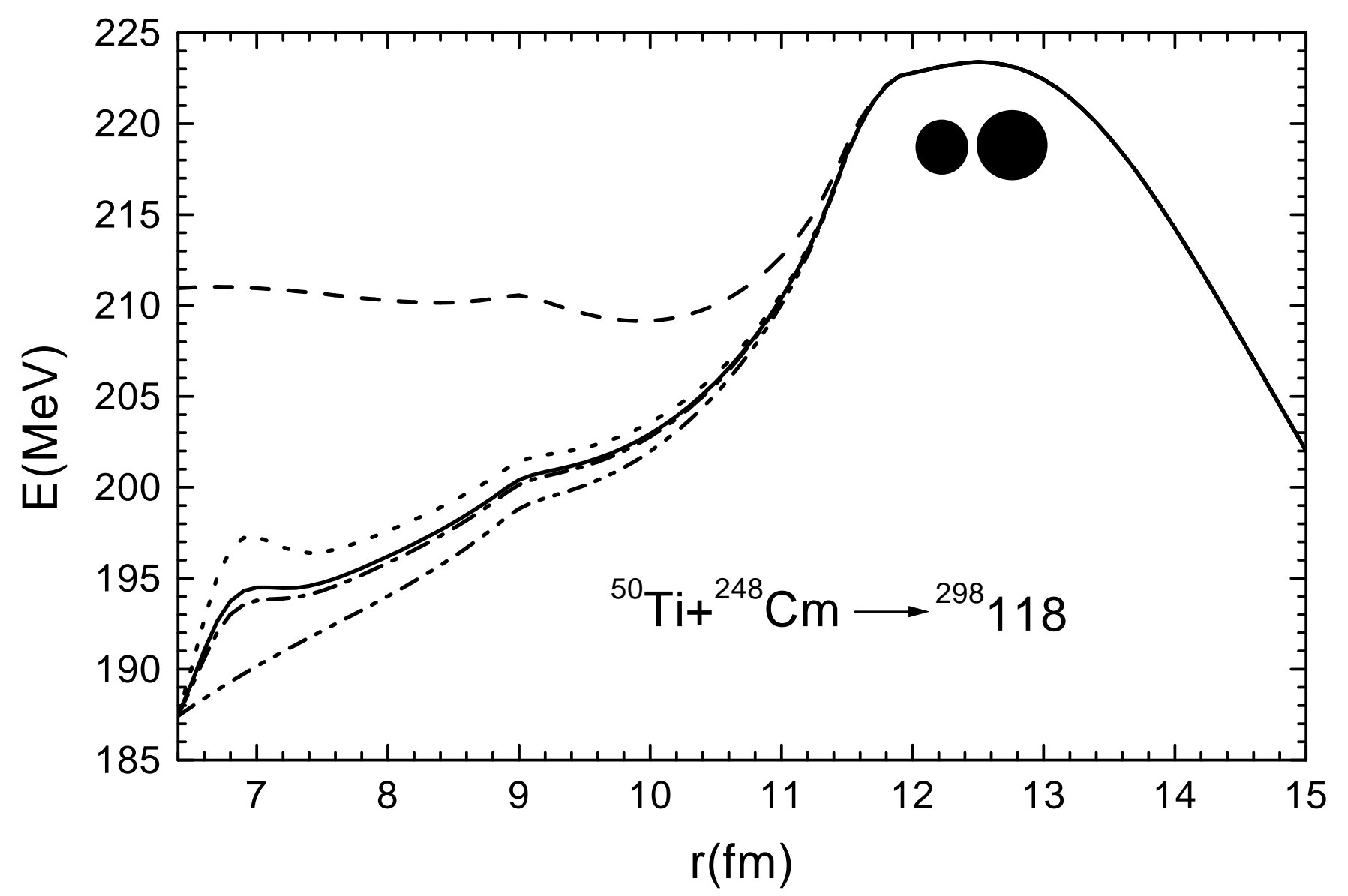




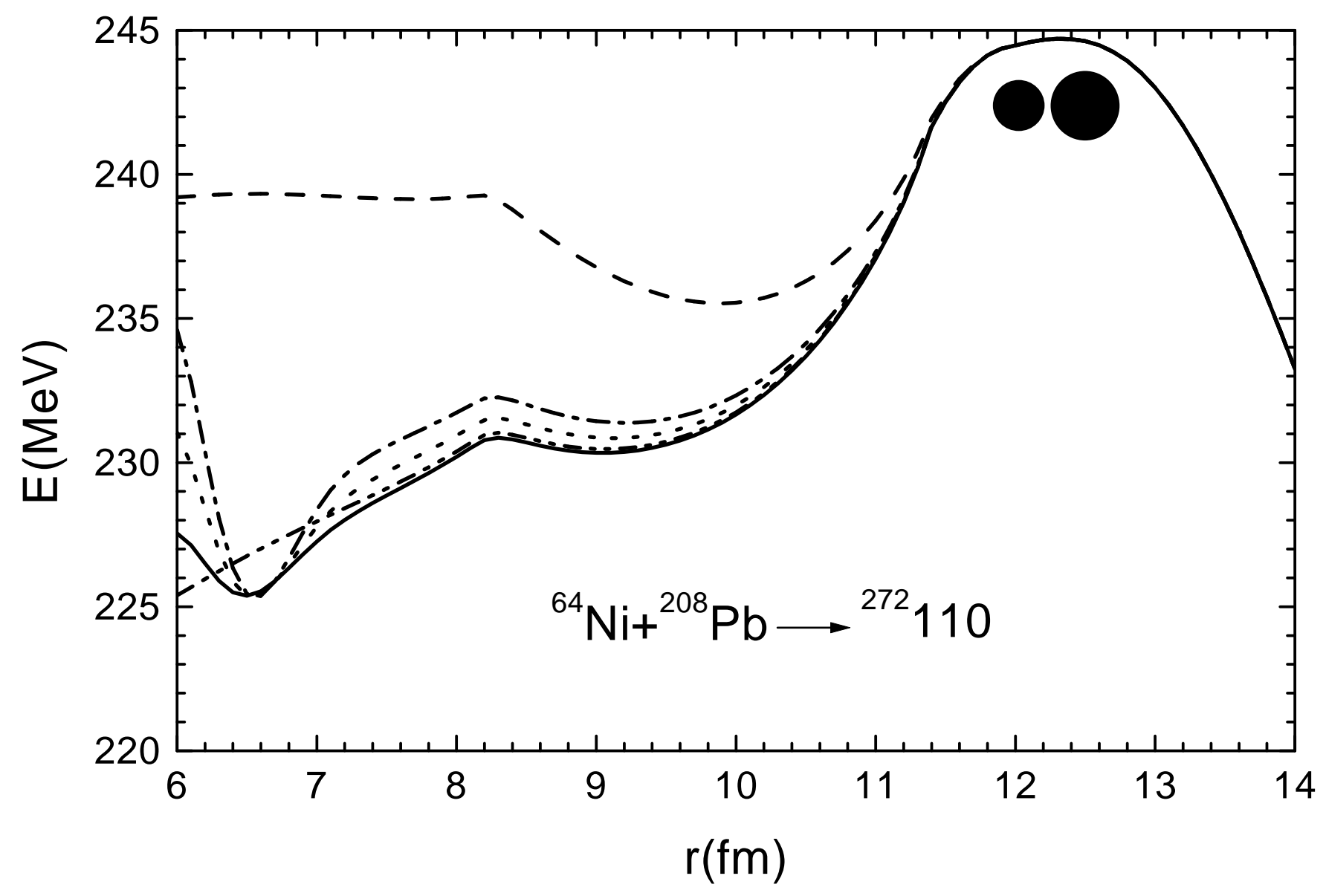




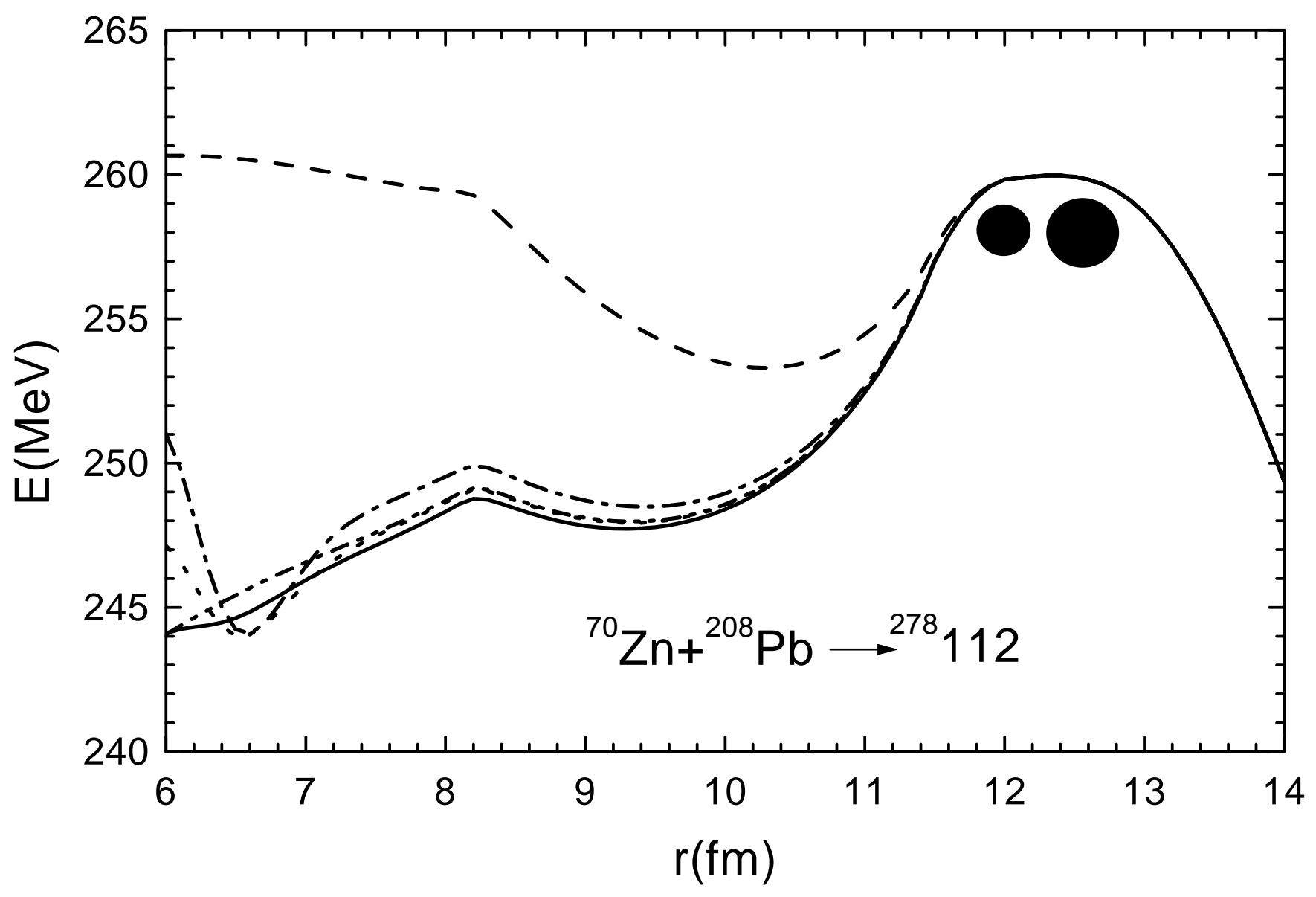




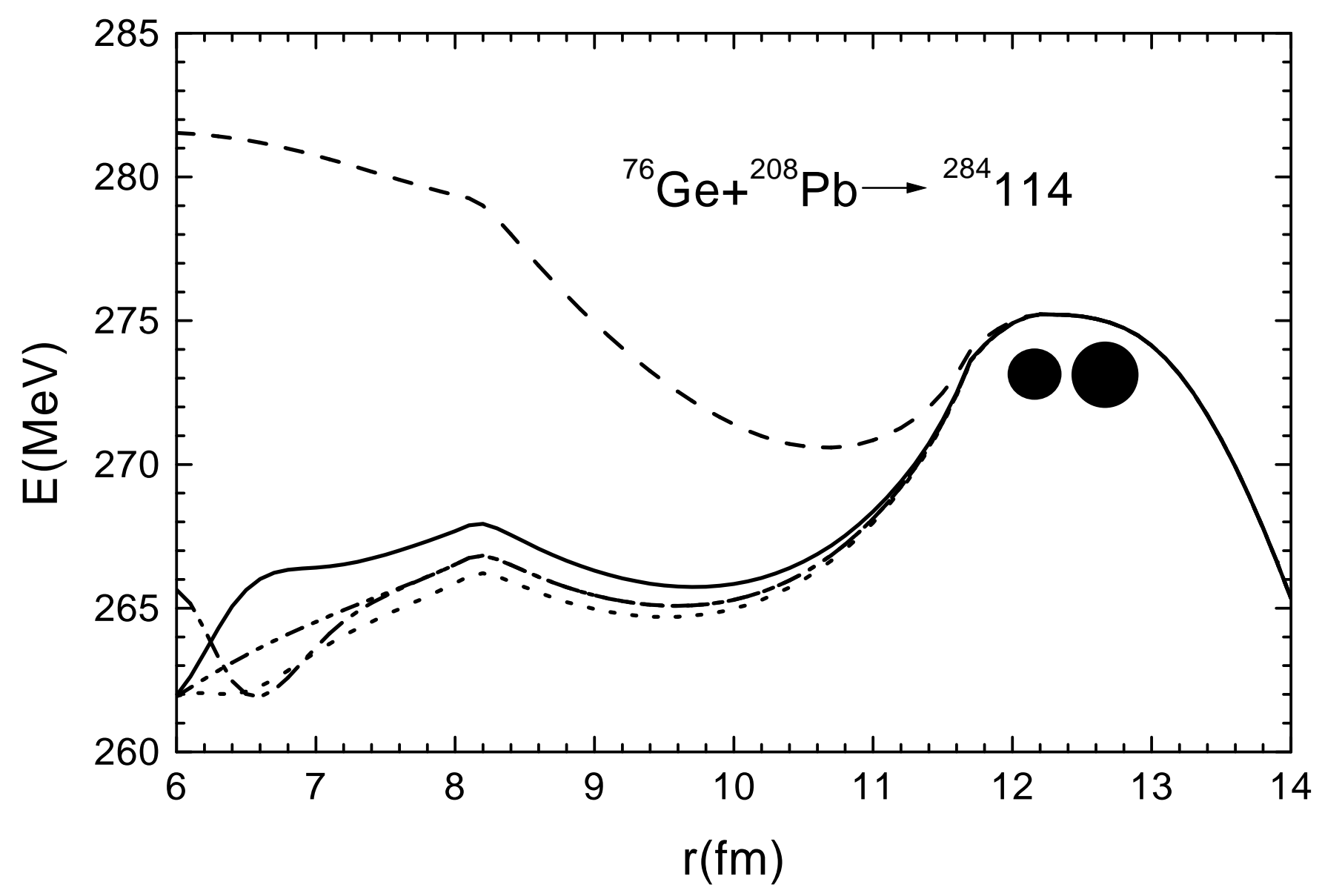




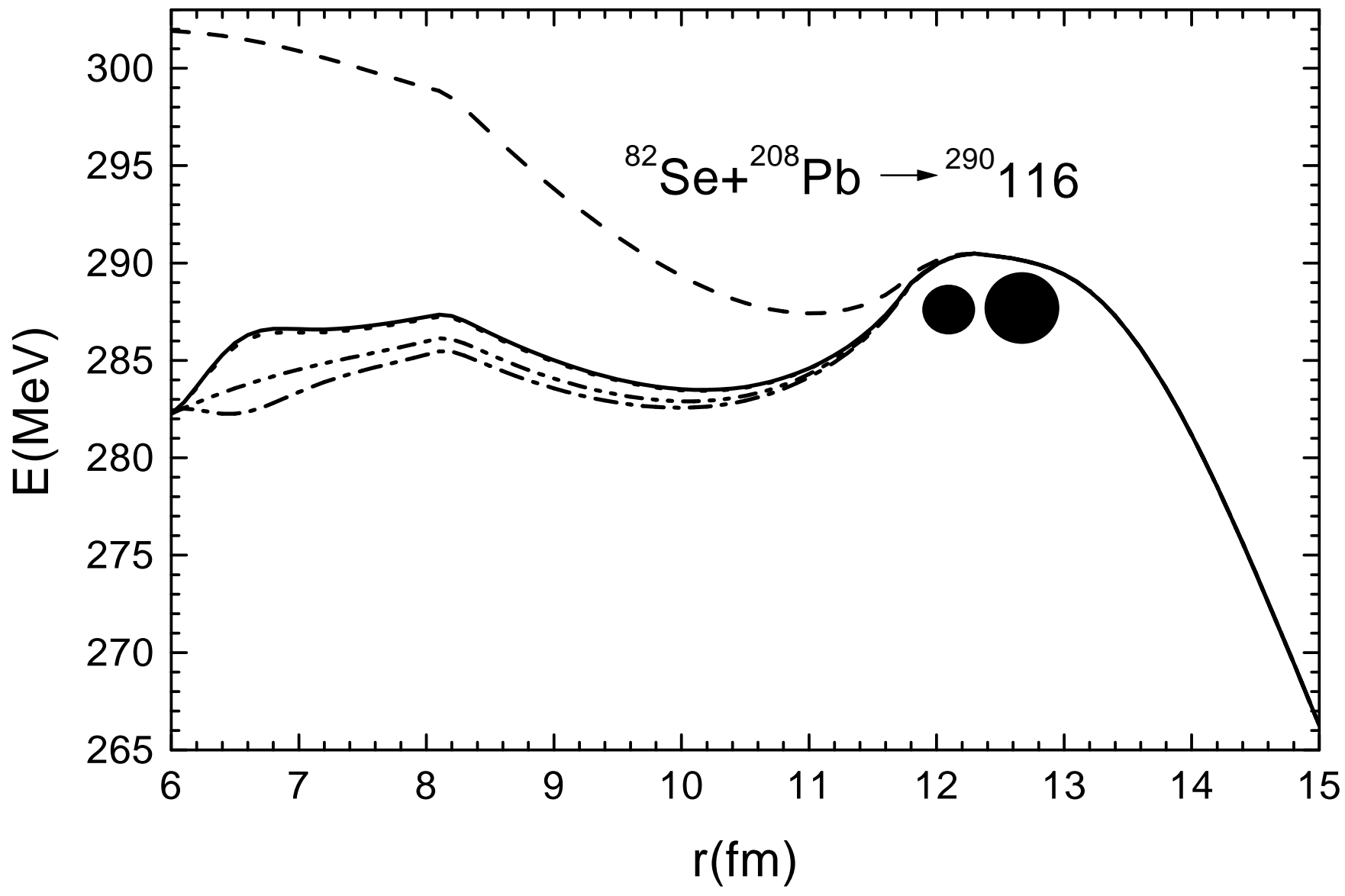




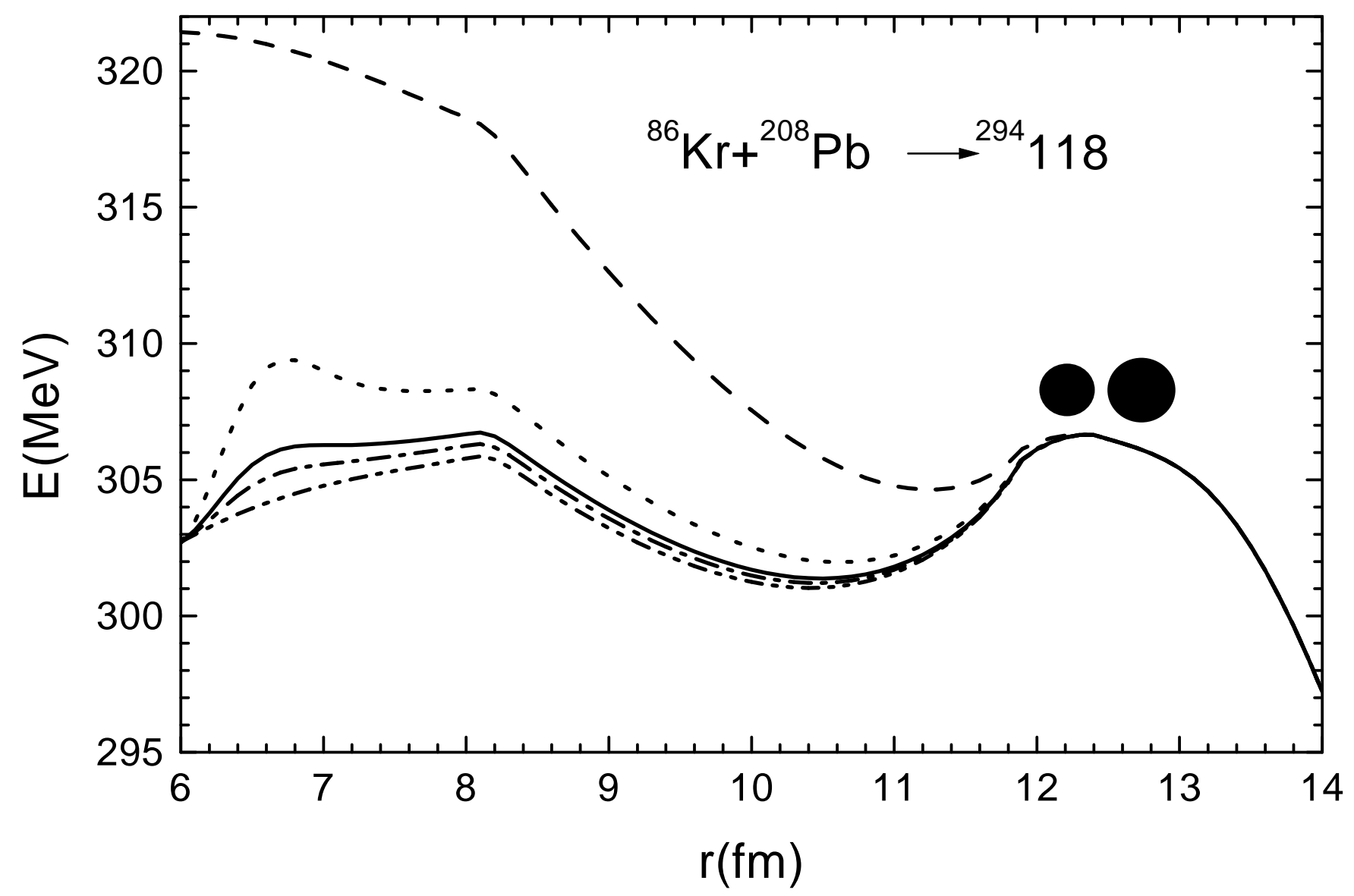




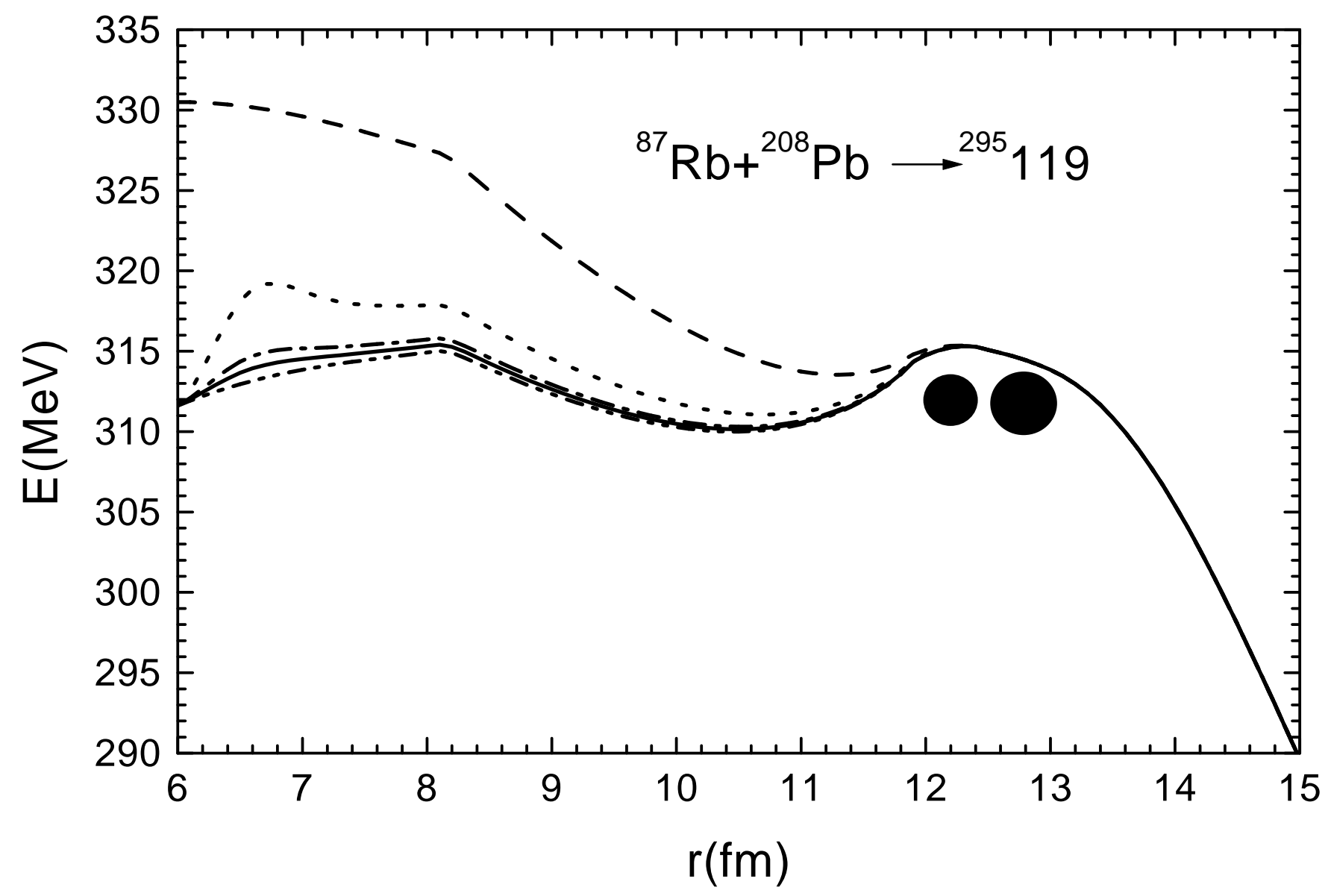




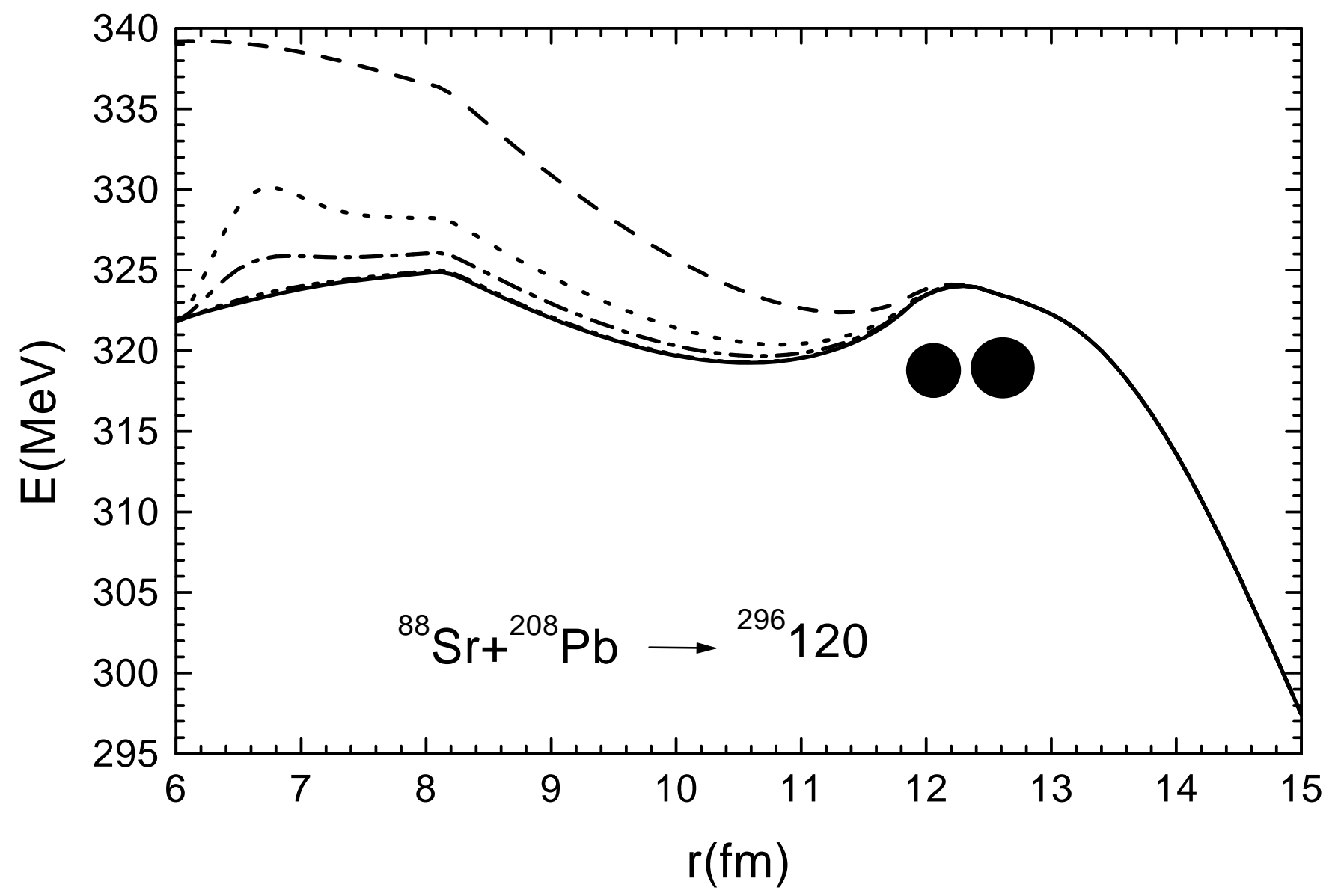




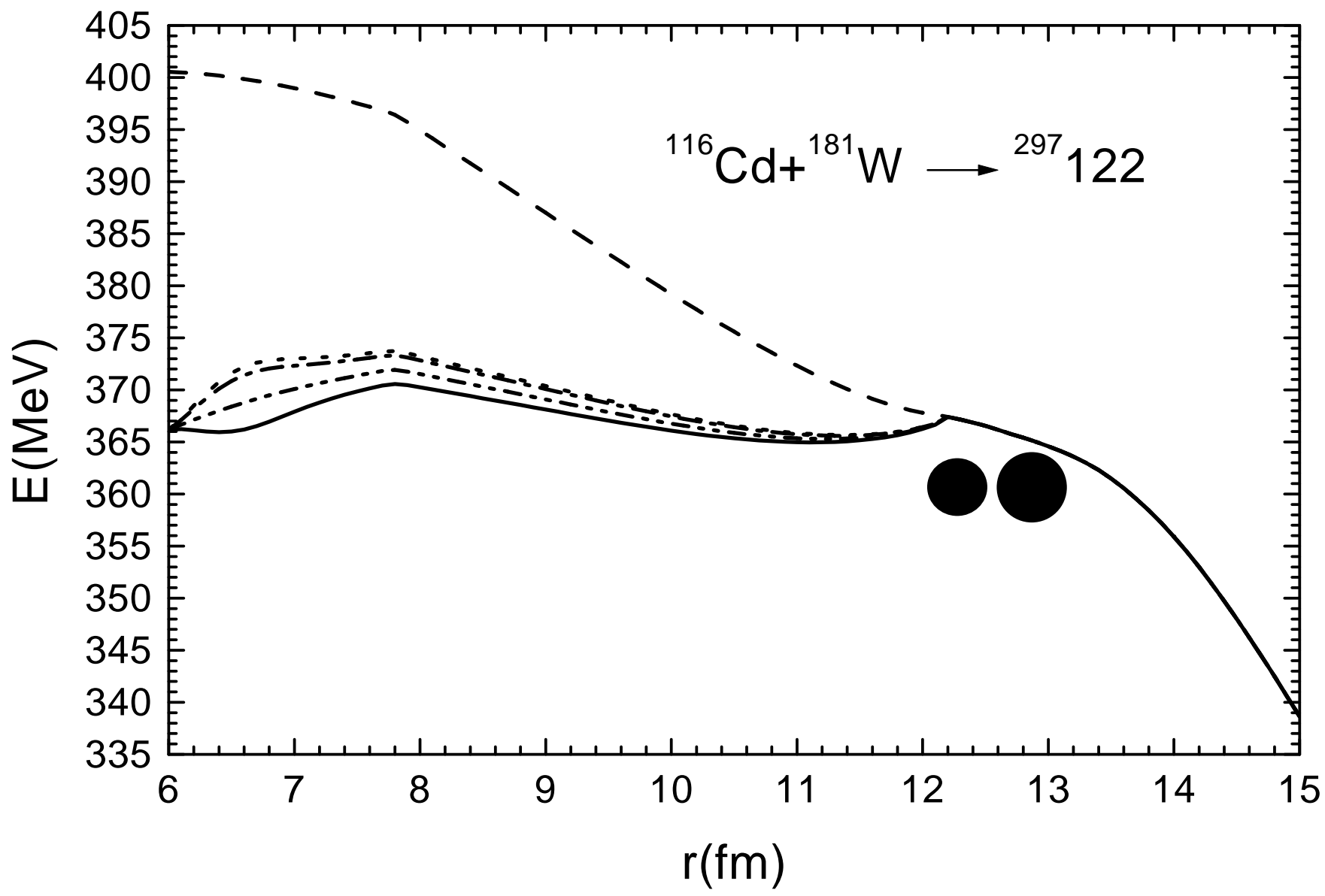




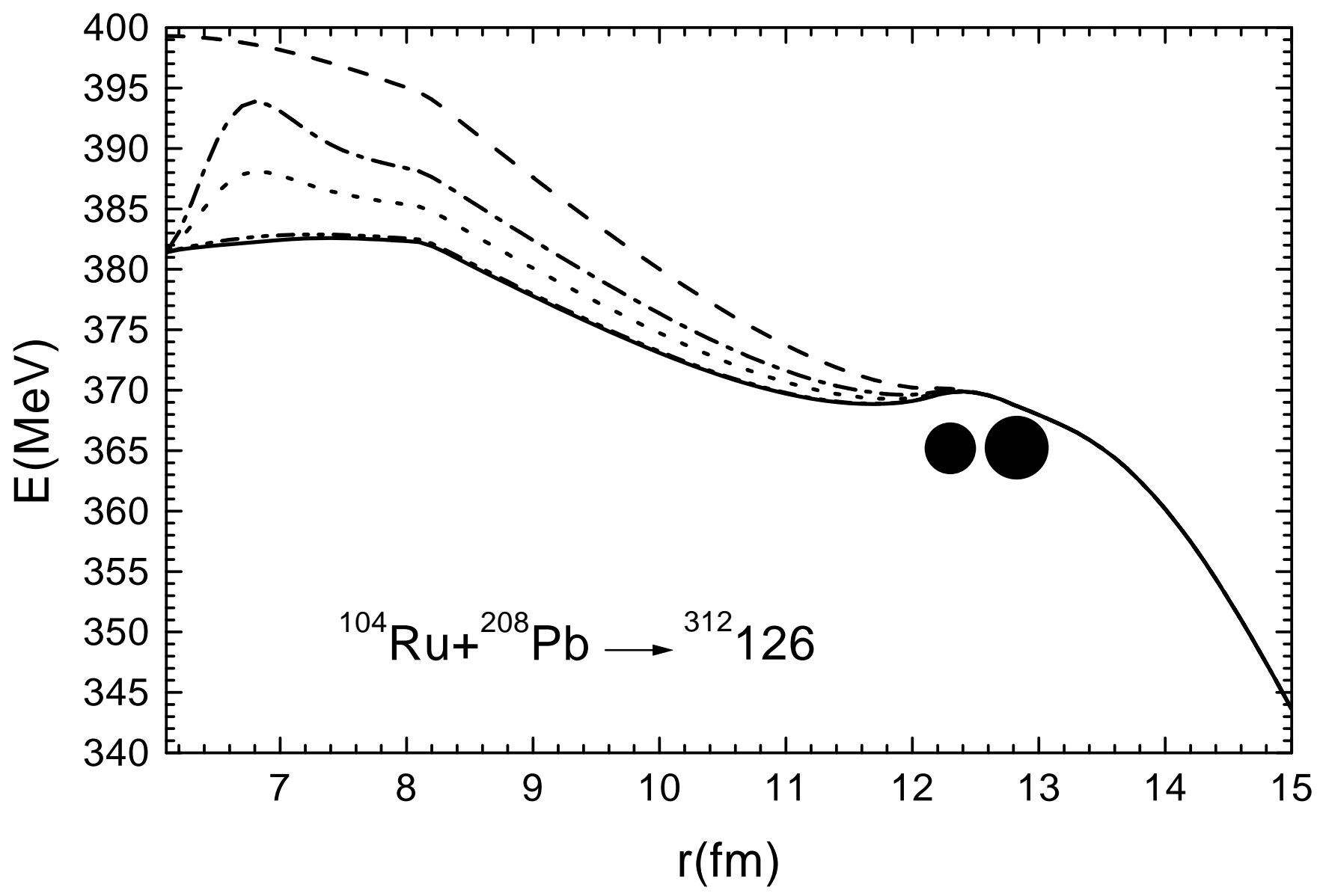




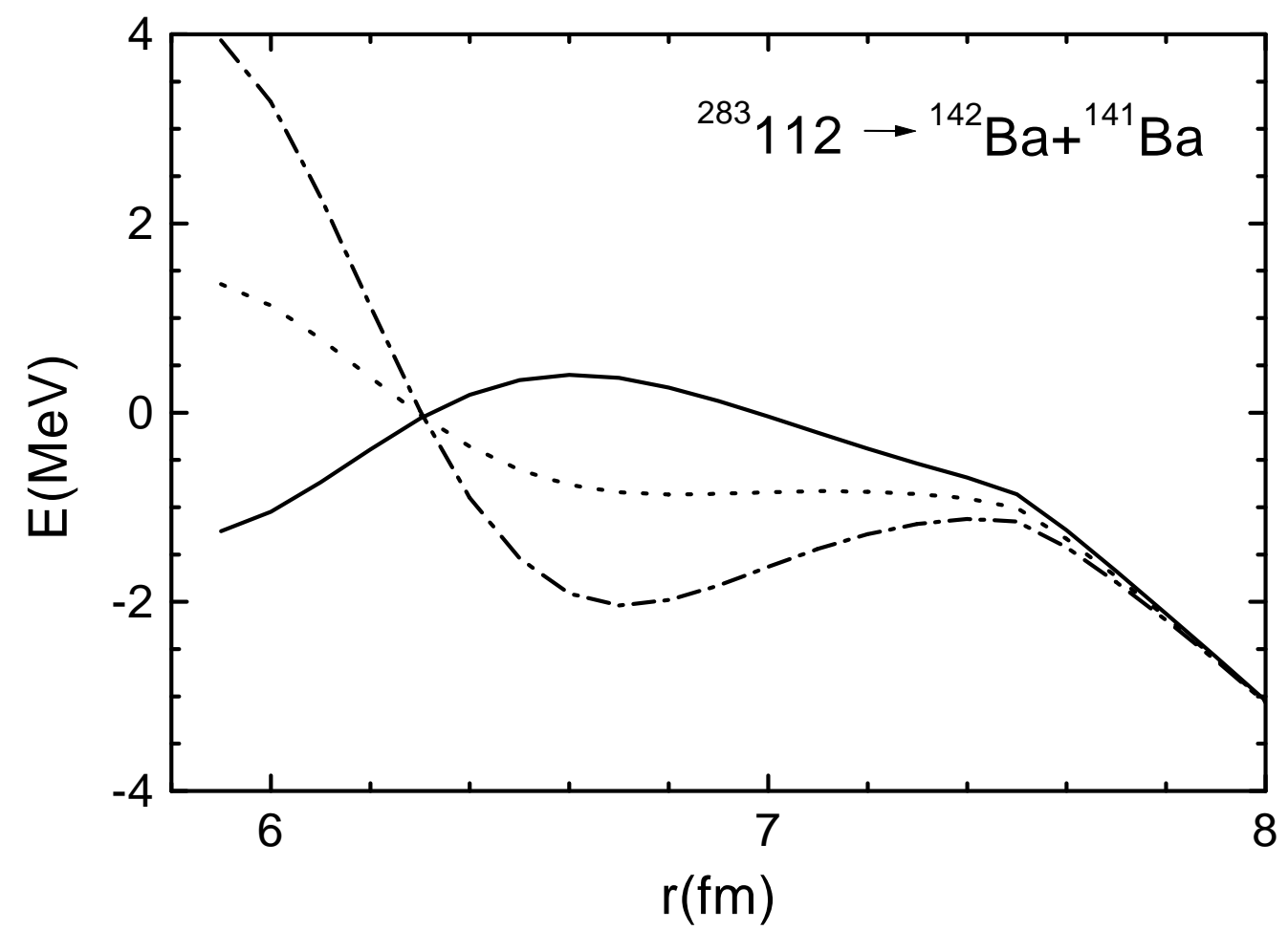




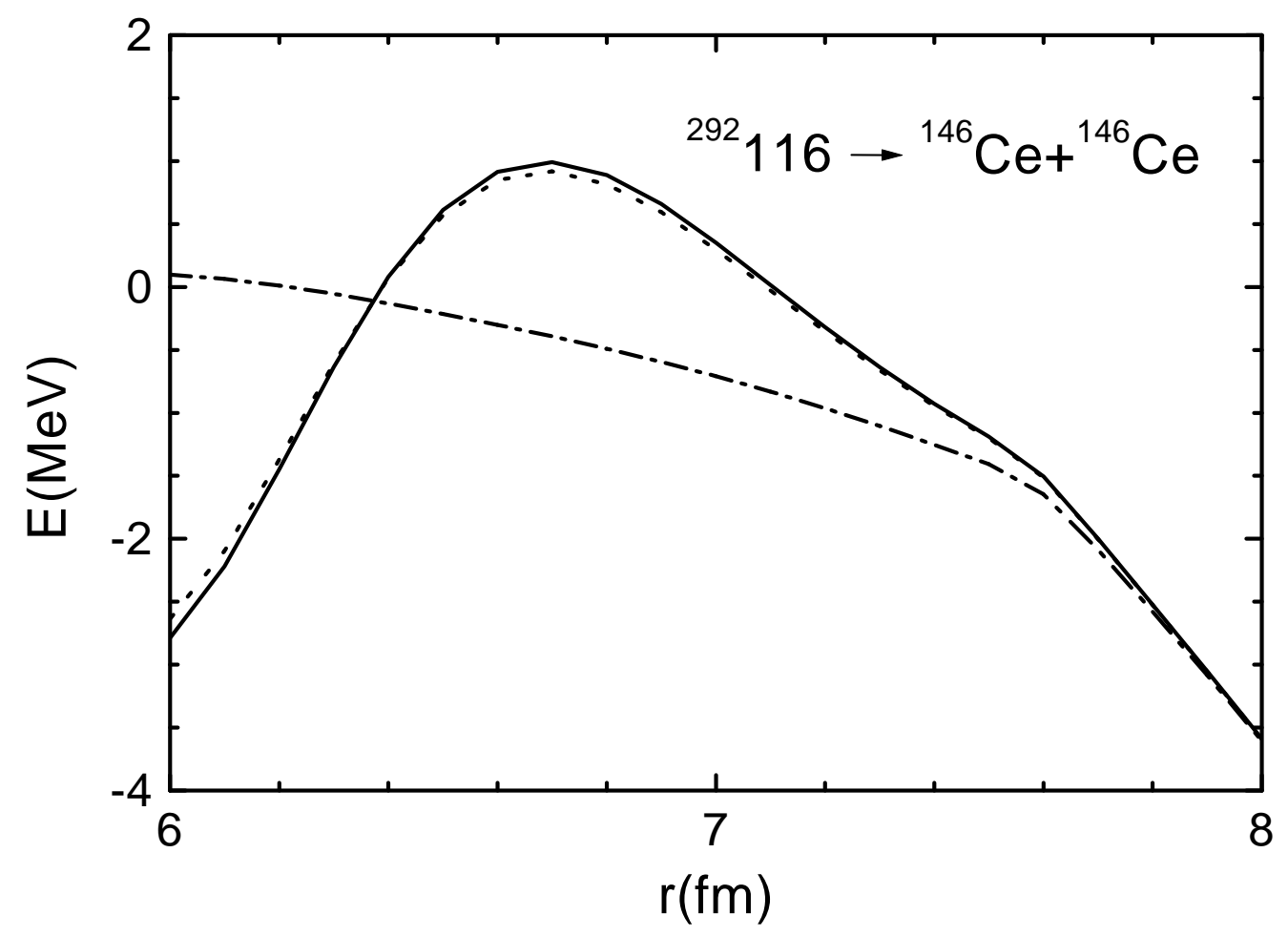




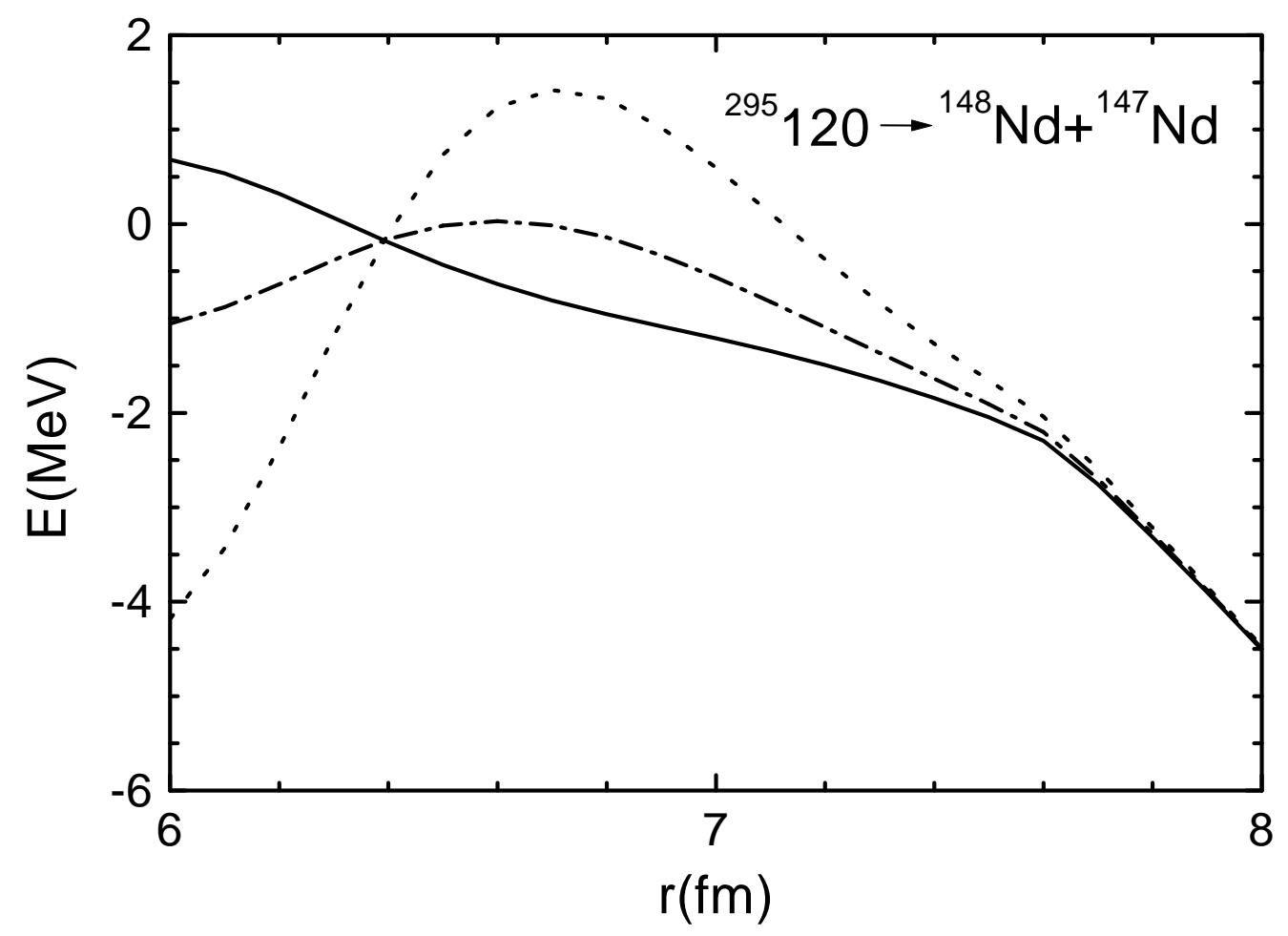




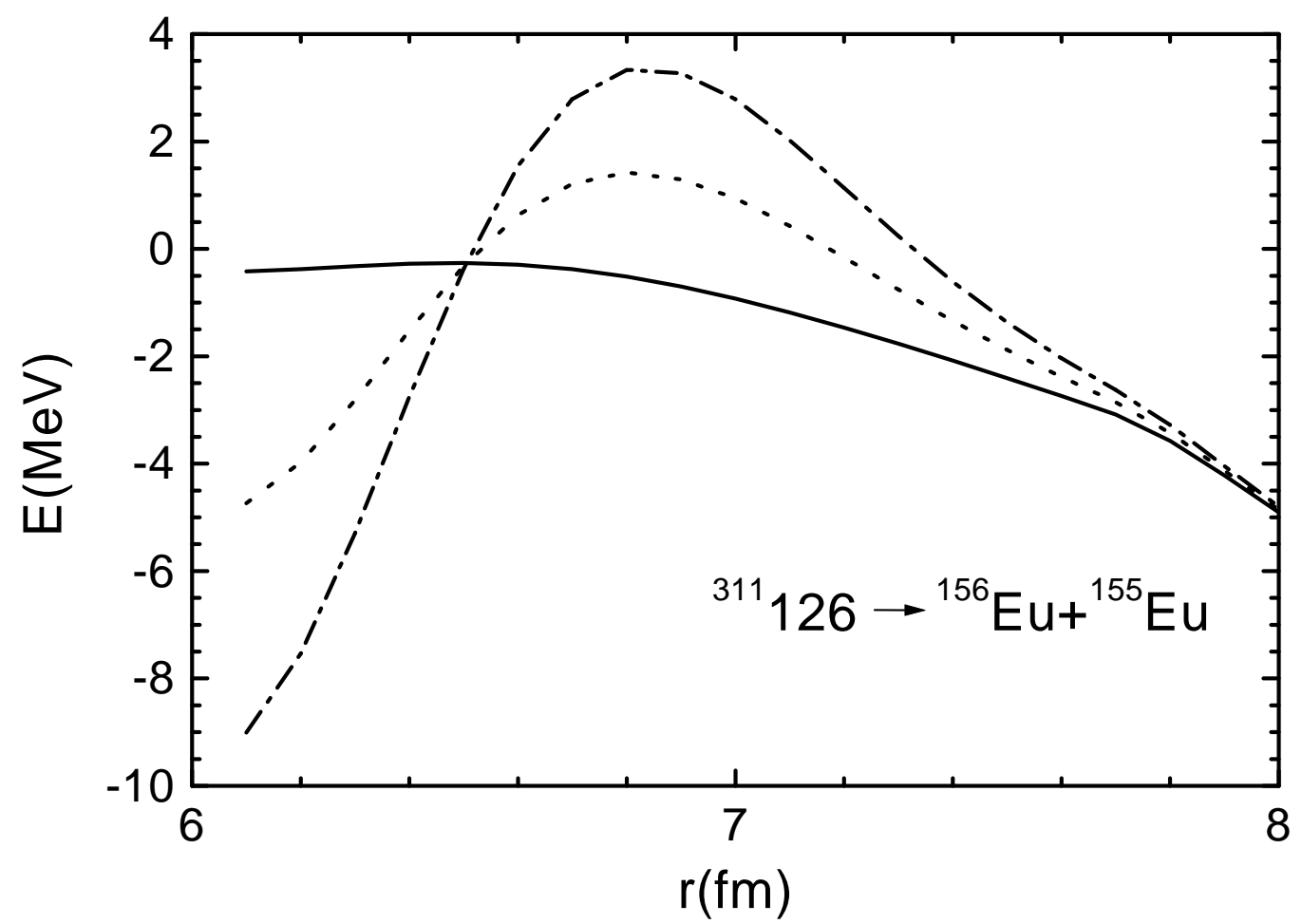

